\title{
Splitting in solvable groups of finite Morley rank
}

\author{
OLIVIER FRÉCON
}

\begin{abstract}
We exhibit counterexamples to a Conjecture of Nesin, since we build a connected solvable group with finite center and of finite Morley rank in which no normal nilpotent subgroup has a nilpotent complement.

The main result says that each centerless connected solvable group $G$ of finite Morley has a normal nilpotent subgroup $U$ and an abelian subgroup $T$ such that $G=U \rtimes T$, if and only if, for any field $K$ of finite Morley rank, the connected definable subgroups of $K^{*}$ are pseudo-tori.
\end{abstract}

Also we build a centerless connected solvable group $G$ of finite Morley rank with no definable representation over a direct sum of interpretable fields.

2000 Mathematics Subject Classification 03C45, 20A15 (primary); 03C60 (secondary)

Keywords: conjecture of Nesin, pseudo-torus, field of finite Morley rank

\section{Introduction}

The main question about groups of finite Morley rank is the Algebraicity Conjecture, which states that the infinite simple ones are isomorphic as abstract groups with an algebraic group over an algebraically closed field. It was independently formulated by Cherlin and Zil'ber in the 70's. This conjecture motivated the search for analogues in groups of finite Morley rank of classical theorems about affine algebraic groups. In this vein, a central question concerning solvable groups of finite Morley rank is a Conjecture of Nesin. It states that the connected ones decompose as $G=U \rtimes T$ for some normal nilpotent definable subgroup $U$ and an abelian subgroup $T$ (see [8, Conjecture p.687] and [3, Section 9.3]). We recall that the term connected for a group $G$ of finite Morley rank means that $G$ is equal to its connected component $G^{\circ}$, which is the smallest definable subgroup of finite index. The first result of the present paper shows that this conjecture fails, even for groups with finite center (Proposition 2.1). Our counterexample is a central product of two algebraic groups, with finite intersection; the intersection is unipotent in one group and semisimple in the other. 
In $\S 3$ and $\S 4$, we consider some weak forms of the Nesin Conjecture, and their restriction to centerless groups. In particular, we study the following questions.

Question 1.1 (1) Do connected solvable groups of finite Morley rank have a decomposition as a product of a normal nilpotent subgroup by an abelian subgroup ?

(2) Does the Nesin Conjecture hold for centerless groups?

Moreover, we notice that, in contrast with Proposition 2.1 below, Wagner proved that any connected solvable group $G$ of finite Morley rank is a product of two connected definable nilpotent subgroups $U$ and $C$ with $U$ normal in $G$ [11]. However, no information is given about the size of the intersection $U \cap C$, and we will consider this question too (see the second part of Theorem 4.2).

Actually, we will show that these questions are related to the structure of fields of finite Morley rank. More precisely, first we state the following conjecture, where pseudo-tori are analogs of tori in algebraic groups introduced in [7], and are defined as abelian divisible groups of finite Morley rank with no definable quotient definably isomorphic to $K_{+}$for any interpretable field $K$.

Conjecture 1.2 For any field $K$ of finite Morley rank, the connected definable subgroups of $K^{*}$ are pseudo-tori.

We notice that the main result of [12] implies that Conjecture 1.2 holds in positive characteristic. The main theorem of this paper is proven in the third section and in the more technical fourth section, and it concerns the link between Question 1.1 and Conjecture 1.2.

Theorem 1.3 (1) (Proposition 3.6) If Conjecture 1.2 holds, then any connected solvable group $G$ of finite Morley rank decomposes as $G=U T$ for a normal nilpotent connected definable subgroup $U$ and a divisible abelian subgroup $T$ such that $U \cap T$ is finite and central in $G$.

(2) (Theorem 4.2) On the other hand, if Conjecture 1.2 does not hold, then there is a centerless connected solvable group $G$ of finite Morley rank satisfying:

(a) $G$ has no decomposition as a product of a normal nilpotent subgroup by an abelian subgroup;

(b) $G$ has no decomposition as a product of a normal nilpotent subgroup $U$ by another nilpotent subgroup $C$ such that $U \cap C$ is finite. 
The conjugacy of the complements will be considered in $\S 3$, and we will show that there is a centerless connected solvable group $G$ of finite Morley rank such that no normal nilpotent subgroup $U$ with nilpotent quotient $G / U$ has the conjugacy of its complements in $G$ (Proposition 3.7). Also, the definability of the complements will be considered in $\S 5$, and we will see that a centerless connected solvable group $G$ of finite Morley rank can have no decomposition of the form $G=U \rtimes T$ for $U$ a normal nilpotent subgroup and $T$ a definable nilpotent subgroup (Proposition 5.1)

On the other hand, Poizat showed in the 80's that, if an infinite simple group $G$ is isomorphic as abstract group with an algebraic group over an algebraically closed field, then we may define in the pure group $G$ an algebraically closed field $K$ and an isomorphism from $G$ to an algebraic group over $K$ [10]. In view of this situation, the linearity and the definable linearity of groups of finite Morley rank have been continuing concerns. Notably, among the most recent results, we may notice the construction of a torsion-free centerless solvable group of finite Morley rank [2], that is a solvable group of finite Morley rank not isomorphic as abstract group with a direct sum of algebraic groups over algebraically closed fields. Also, it is now known that all the torsion-free nilpotent groups of finite Morley rank are linear [1]. We will conclude this paper by counterexamples to some natural questions on linearity. In particular, we build a centerless connected solvable group $G$ of finite Morley rank with no definable representation over a direct sum of fields (Proposition 5.3).

\section{A counterexample to a Conjecture of Nesin}

If $G$ is a connected solvable algebraic group, then $G=U \rtimes T$ where $U$ is the unipotent radical and $T$ any maximal torus. In view of this theorem, Nesin conjectured that any connected solvable group of finite Morley rank has a similar decomposition, namely there is a nilpotent definable subgroup with an abelian complement (see [8, Conjecture p.687] and [3, Section 9.3]). In this section, we provide a counterexample to this conjecture.

We recall that the Fitting subgroup $F(G)$ of any group $G$ is the subgroup generated by all its normal nilpotent subgroups. Note that, in any group $G$ of finite Morley rank $F(G)$ is definable and nilpotent [3, Theorem 7.3].

Proposition 2.1 There exists a connected solvable group $G$ of finite Morley rank with finite center and such that no normal nilpotent subgroup has a nilpotent complement. 
Proof Let $K$ and $L$ be two algebraically closed fields of characteristic $p$ and $q$ respectively, where $p$ is a prime and $q \neq p$ is either a prime or zero. We consider

$$
A_{0}=\left\{\left(\begin{array}{ccc}
1 & a & b \\
0 & r & c \\
0 & 0 & 1
\end{array}\right) \mid r \in K^{*},(a, b, c) \in K \times K \times K\right\} .
$$

Then the center of $A_{0}$ is $Z\left(A_{0}\right)=\left\{\left(\begin{array}{lll}1 & 0 & b \\ 0 & 1 & 0 \\ 0 & 0 & 1\end{array}\right) \mid b \in K\right\}$, and it has an element $x$ of order $p$. The quotient of $A_{0}$ by $\langle x\rangle$ is an algebraic group over $K$, and it acts faithfully by multiplication on $K^{n}$ for a positive integer $n$. From now on, we consider the group $A=K^{n} \rtimes\left(A_{0} \times K^{*}\right)$ where, for each $(a, k) \in A_{0} \times K^{*}$ and each $u \in K^{n}$, the action of $(a, k)$ over $u$ is defined by the one of the image $\bar{a}$ of $a$ in $A_{0} /\langle x\rangle$ over $u$ and by the scalar multiplication of $k$ over $u$. In particular, the center of $A$ is $\langle x\rangle$.

Now let $B$ be a Borel subgroup of $\operatorname{SL}(p, L)$, and $U_{B}$ its unipotent radical. Then the center $Z_{B}$ of $\operatorname{SL}(p, L)$ is cyclic of order $p$, it is contained in each maximal torus of $B$, and $F(B)=U_{B} Z_{B}$. Let $y$ be a nontrivial element of $Z_{B}$, let $G_{0}=A \times B$, and let $H=\left\langle x y^{-1}\right\rangle$. Then $H$ is a central subgroup of $G_{0}$, and it is cyclic of order $p$. We consider the pure group $G_{0}$. Since $K$ and $L$ are algebraically closed, $G_{0}$ has finite Morley rank, and $\bar{G}=G_{0} / H$ is a solvable connected definable quotient. Moreover, since the center of $G_{0}$ is $\langle x, y\rangle$, which is a finite subgroup, the center of $\bar{G}$ is $\langle x, y\rangle / H$, and it is finite too.

We assume toward a contradiction that $\bar{G}$ has a normal nilpotent subgroup $\bar{U}=U_{0} / H$ and a nilpotent subgroup $\bar{T}=T_{0} / H$ such that $\bar{G}=\bar{U} \rtimes \bar{T}$. First we show that $U_{0}=F\left(G_{0}\right)$. Since $H \leq Z\left(G_{0}\right)$, the subgroup $U_{0}$ is nilpotent and contained in $F\left(G_{0}\right)=F(A) \times F(B)$. On the other hand, since $\bar{T}$ is nilpotent, $G / U_{0}$ is nilpotent too. We notice that $F(A)=K^{n} \rtimes F\left(A_{0}\right)$, where

$$
F\left(A_{0}\right)=\left\{\left(\begin{array}{lll}
1 & a & b \\
0 & 1 & c \\
0 & 0 & 1
\end{array}\right) \mid(a, b, c) \in K \times K \times K\right\} .
$$

In particular, we have $\left[A_{0}, F\left(A_{0}\right)\right]=F\left(A_{0}\right)$, and $F\left(A_{0}\right)$ is the only normal nilpotent subgroup of $A_{0}$ such that $A_{0} / F\left(A_{0}\right)$ is nilpotent. Then, if $N_{A}$ denotes a normal nilpotent subgroup of $A$ such that $A / N_{A}$ is nilpotent, the groups $N_{A} \cap A_{0}$ and $A_{0} /\left(N_{A} \cap A_{0}\right)$ are nilpotent, so $N_{A} \cap A_{0}=F\left(A_{0}\right)$. In the same way, the Fitting subgroup of $K^{n} \rtimes K^{*} \leq A$ is $K^{n}$ and we have $\left[K^{n}, K^{*}\right]=K^{n}$ since $K^{*}$ acts by multiplication on $K^{n}$, so $K^{n}$ is the only normal nilpotent subgroup of $K^{n} \rtimes K^{*}$ such that $\left(K^{n} \rtimes K^{*}\right) / K^{n}$ is nilpotent. This implies that $N_{A} \cap\left(K^{n} \rtimes K^{*}\right)=K^{n}$, therefore we obtain $N_{A}=K^{n} \rtimes F\left(A_{0}\right)=F(A)$. 
Thus, since $G / U_{0}$ is nilpotent, we obtain $F(A) \leq U_{0}$ and $x \in U_{0}$. Moreover, we have $\left[B, U_{B}\right]=U_{B}$, so $U_{B}$ is the only normal nilpotent subgroup of $B$ such that $B / U_{B}$ is nilpotent. Therefore, since $G / U_{0}$ is nilpotent, $U_{0}$ contains $U_{B}$. Now, since $x \in U_{0}$ and since $H=\left\langle x y^{-1}\right\rangle$ is contained in $U_{0}$, we have $Z_{B}=\langle y\rangle \leq U_{0}$, and the subgroup $F(B)=U_{B} Z_{B}$ is contained in $U_{0}$. This proves that $U_{0}=F\left(G_{0}\right)$.

We show that $x$ belongs to $T_{0}$. Since $U_{0}=F\left(G_{0}\right)=F(A) \times F(B)$, the group $\bar{T}$ is isomorphic to $G_{0} / U_{0} \simeq A / F(A) \times B / F(B)$. In particular, it is abelian and divisible. Thus $\bar{T}$ has a unique maximal $p$-subgroup $\bar{S}=S_{0} / H$, and this subgroup is abelian and divisible. Moreover, since $H$ is a $p$-subgroup, $S_{0}$ is a $p$-subgroup too. But $H$ is central in $G_{0}$ hence, for each $g \in S_{0}$, the map $\operatorname{ad}_{g}: S_{0} \rightarrow H$, defined by $\operatorname{ad}_{g}(x)=[g, x]$, is a homomorphism and $\operatorname{ad}_{g}\left(S_{0}\right) \simeq S_{0} / C_{S_{0}}(g)$ is divisible. Since $H$ is finite, this proves that $C_{S_{0}}(g)=S_{0}$ for each $g \in S_{0}$, therefore $S_{0}$ is abelian and, by divisibility of $S_{0} / H$, the set $R_{0}=\left\{g^{p^{2}} \mid g \in S_{0}\right\}$ is a subgroup of $S_{0}$ such that $S_{0}=R_{0} H$. Also $R_{0}$ is divisible, since it is the image of the homomorphism $\alpha: S_{0} \rightarrow S_{0}$ defined for each $g \in S_{0}$ by $\alpha(g)=g^{2}$, and $H$ lies in the kernel of $\alpha$. Since the characteristic of $K$ is $p$, all the $p$-elements of $A$ are unipotent, so they are contained in $F(A)$, and $A / F(A)$ has no nontrivial $p$-element. On the other hand, $F(A)$ is a $p$-group of exponent at most $p^{2}$, so $R_{0}$ is contained in $B$. Since the characteristic of $L$ is $q \neq p$, each divisible abelian $p$-subgroup of $B$ is contained in a maximal torus of $B$, and $R_{0}$ is contained in a maximal torus $T_{B}$ of $B$. Let $R_{B}$ be the maximal $p$-subgroup of $T_{B}$. Then $R_{B}$ contains $R_{0}$ and, since $R_{B}$ is isomorphic to the maximal $p$-subgroup of $\left(L^{*}\right)^{p-1}$, that is its Prüfer rank is finite, we have either $R_{B}=R_{0}$, or $R_{B}$ is not isomorphic to $R_{0}$. Since $U_{0}=F(A) \times F(B)$, the subgroup $\bar{T}$ is isomorphic to $A / F(A) \times B / F(B)$ and, since $A / F(A)$ has no nontrivial $p$-element, $\bar{S}$ is isomorphic to the unique maximal $p$-subgroup of $B / F(B) \simeq\left(L^{*}\right)^{p-1}$. This proves that $R_{0}$ is isomorphic to $R_{B}$, and we obtain $R_{B}=R_{0}$. But $y$ is a central semisimple element of $B$, so it is contained in $T_{B}$, and $R_{0}=R_{B}$ contains $\langle y\rangle$. In particular we obtain $y \in T_{0}$ and, since $x y^{-1} \in H$ belongs to $T_{0}$, we have $x \in T_{0}$.

Therefore, we have $x \in F(A) \leq U_{0}$ and $x \in T_{0}$, so $x \in U_{0} \cap T_{0}=H$ contradicting the choice of $H$. This finishes the proof.

\section{In the case where Conjecture 1.2 holds}

In this section, we prove the first part of Theorem 1.3 (Proposition 3.6). Our analysis depends on the following four results. 
On the other hand, we provide a counterexample to a natural question concerning the conjugacy of some complements in connected solvable groups of finite Morley rank (Proposition 3.7).

Fact 3.1 [3, Theorem 9.21] Let $G$ be a connected and solvable group of finite Morley rank. Then $G / F(G)^{\circ}$ (so also $G / F(G)$ ) is a divisible abelian group.

As in [3], in any group $G$ of finite Morley rank and for any subset $X$ of $G$, a definable subgroup $A$ is said to be $X$-minimal if it is infinite, normalized by $X$ and minimal for these conditions.

Fact 3.2 [3, Theorem 9.1] Let $G=A \rtimes H$ be a group of finite Morley rank where $A$ and $H$ are two infinite definable abelian subgroups, $A$ is $H$-minimal and $C_{H}(A)=1$. Then $G$ interprets an algebraically closed field $K$ such that $A \simeq K_{+}$definably, and such that $H$ is definably isomorphic to a subgroup of $K^{*}$.

Fact 3.3 [7, Corollary 2.8] Any nilpotent group of finite Morley rank has a unique maximal pseudo-torus.

Fact 3.4 [7, Corollary 2.13] Let $G$ be a group of finite Morley rank, $N$ a normal definable subgroup of $G$ and $T$ a maximal pseudo-torus of $G$. Then $T N / N$ is a maximal pseudo-torus of $G / N$.

Fact 3.5 [5, Lemma 4.20] Let $G$ be a solvable connected group of finite Morley rank and let $T$ be an abelian divisible $p$-subgroup for a prime $p$. Then $T \cap F(G)$ is contained in $Z(G)$.

Proposition 3.6 Suppose that Conjecture 1.2 holds, and let $G$ be a connected solvable group of finite Morley rank. Then $G=F(G)^{\circ} T$ for $T$ a divisible abelian subgroup of $G$, such that $F(G) \cap T$ is finite and central in $G$.

In particular, if $G$ is centerless, then $F(G)$ is connected and $G=F(G) \rtimes T$.

Proof Let $P$ be a maximal pseudo-torus of $G$. We show that $G=F(G)^{\circ} P$. Since $F(G)$ is nilpotent, it is the intersection of the centralizers of $G$-minimal sections in $G$, and we find finitely many $G$-minimal sections $\overline{A_{1}}, \ldots, \overline{A_{n}}$ of $G$ such that $F(G)=C_{G}\left(\overline{A_{1}}, \cdots, \overline{A_{n}}\right)$. On the other hand, for each $G$-minimal section $\bar{A}$ of $G$, the quotient $G / C_{G}(\bar{A})$ is abelian by Fact 3.1, and Fact 3.2 says that each connected definable subgroup of $G / C_{G}(\bar{A})$ is a pseudo-torus since Conjecture 1.2 holds. Then, 
by considering $C_{0}=G$ and $C_{i}=C_{G}\left(\overline{A_{1}}, \cdots, \overline{A_{i}}\right)$ for each $i \in\{1, \ldots, n\}$, since $C_{i} / C_{i+1}=C_{i} /\left(C_{i} \cap C_{G}\left(\overline{A_{i+1}}\right)\right)$ is definably isomorphic to $C_{i} C_{G}\left(\overline{A_{i+1}}\right) / C_{G}\left(\overline{A_{i+1}}\right) \leq$ $G / C_{G}\left(\overline{A_{i+1}}\right)$ for each $i \in\{0, \ldots, n-1\}$, the group $C_{i}^{\circ} C_{i+1} / C_{i+1}$ is a pseudo-torus for each $i \in\{0, \ldots, n-1\}$. Thus, since we have $F(G)=C_{n}$ and since $G / F(G)$ is abelian (Fact 3.1), we obtain $C_{i}^{\circ} C_{i+1} / C_{i+1} \leq P C_{i+1} / C_{i+1}$ for each $i \in\{0, \ldots, n-1\}$ by Facts 3.3 and 3.4. Consequently $P F(G) / F(G)$ has finite index in $G / F(G)=C_{0} / C_{n}$ and, since $G$ is connected, we find $G=F(G) P=F(G)^{\circ} P$.

Since $P$ is divisible and abelian, $\left(F(G)^{\circ} \cap P\right)^{\circ}$ is divisible and abelian too, so $\left(F(G)^{\circ} \cap\right.$ $P)^{\circ}$ has a complement $T$ in $P$. Then $T$ is a divisible abelian subgroup of $G$ such that $G=F(G)^{\circ} T$, and such that $F(G) \cap T$ is finite. Furthermore, for each prime $p$, the $p$-elements of $T$ are contained in an abelian divisible $p$-subgroup since $T$ is abelian and divisible, so Fact 3.5 says that $F(G) \cap T$ is central in $G$.

By the following remark, we cannot expect the conjugacy of all the subgroups $T$ in Proposition 3.6, even if the ambient group is centerless. Indeed, in some centerless connected solvable groups of finite Morley rank, the Fitting subgroup has definable complements and nondefinable complements. On the other hand, concerning the definability of complements, we will show in $\S 5$ that, in some centerless connected solvable groups of finite Morley rank, the Fitting subgroup has no definable complement.

Proposition 3.7 There is a centerless connected solvable group $G$ of finite Morley rank such that any normal nilpotent subgroup $U$ with nilpotent quotient $G / U$ satisfies:

(1) $U$ is torsion-free;

(2) $U$ has a definable complement;

(3) $U$ has a nondefinable complement.

In particular, the complements of $U$ in $G$ are not all conjugate.

Furthermore, we may choose $G$ to be isomorphic as abstract group with a connected algebraic group over an algebraically closed field.

Proof Let $K$ be an algebraically closed field of characteristic zero and let

$$
G=\left\{\left(\begin{array}{cccc}
x & a_{1} & a_{2} & a_{3} \\
0 & 1 & a_{4} & a_{5} \\
0 & 0 & y & a_{6} \\
0 & 0 & 0 & 1
\end{array}\right) \mid(x, y) \in K^{*} \times K^{*}, a_{i} \in K \text { for } i=1, \cdots, 6\right\} .
$$


We consider the group $G$ in the pure field $K$. Then $G$ is a centerless connected solvable group of Morley rank 8, and $F(G)$ is the unipotent radical, so $F(G)$ is torsion-free. Moreover, we notice that the definable subsets of $G$ are the constructible ones.

Let $U$ be a normal nilpotent subgroup with nilpotent quotient $G / U$. Then $U$ is contained in $F(G)=G^{\prime}$. On the other hand, we have $\left[G, G^{\prime}\right]=G^{\prime}$. Then, since $G / U$ is nilpotent, we obtain $U=G^{\prime}=F(G)$. In particular, $U$ is the unipotent radical of $G$ and any maximal torus of $G$ is a complement of $U$ in $G$. Thus there exists definable complements of $U$ in $G$.

However, if $T$ denotes a maximal torus of $G$, then $V=C_{U}(T)$ is nontrivial and abelian. Since $V \leq U$ is a definable torsion-free group, it is divisible, and $T V$ is an abelian divisible group too. Then there exists a complement $R$ of $V$ in $T V$ containing a fixed element $x$ in $(T V) \backslash(T \cup V)$. Now, since $U \cap T V=V$ and since $G=U \rtimes T$, the subgroup $R$ is a complement of $U$ in $G$. Nevertheless, $T$ is the unique maximal torus of $T V$ and it does not contain $x$, so $R$ is not a torus. Consequently $R$ is nondefinable, otherwise $R \simeq G / U \simeq T$ would be a torus.

\section{The case where Conjecture 1.2 does not hold}

In this section, we prove the second part of Theorem 1.3 (Theorem 4.2).

We will use Carter subgroups, which are defined as the connected definable nilpotent subgroups of finite index in their normalizer. The following fact gives their two properties useful for the proof of Theorem 4.2.

Fact 4.1 Let $G$ be a connected solvable group of finite Morley rank. Then $G$ satisfies the following properties.

(1) [11] Any two Carter subgroups of $G$ are conjugate.

(2) [4, Results 1.2 and 4.4] If $H$ denotes a maximal proper connected definable subgroup of $G$, then either $H$ is normal in $G$, or $H$ contains a Carter subgroup of $G$.

By the following theorem, if Conjecture 1.2 does not hold, we can build a centerless connected group of finite Morley rank with nonalgebraic properties. In particular, both of the answers to Questions 1.1 (1-2) are negative in this case.

Theorem 4.2 If Conjecture 1.2 does not hold, then there exists a centerless connected solvable group $G$ of finite Morley rank such that: 
(1) $G$ has no decomposition as a product of a normal nilpotent subgroup by an abelian subgroup;

(2) $G$ has no decomposition as a product of a normal nilpotent subgroup $U$ by another nilpotent subgroup $C$ such that $U \cap C$ is finite.

Proof By hypothesis, there is a field $K$ of finite Morley rank with a connected definable subgroup $R$ of $K^{*}$ which is not a pseudo-torus. Then $R$ has a definable subgroup $A$ such that $R / A$ is definably isomorphic to $L_{+}$for an interpretable field $L$. We may assume $L=R / A$, and we denote by $+_{L}$ the addition in $L$ and by ${ }_{L}$ the multiplication. Moreover, we denote by $1_{K} \in R$ the identity element of $K^{*}$, and by $1_{L} \in L$ the one of $L^{*}$. We consider the following group, which can be seen as a slightly bent subgroup of $\operatorname{GL}(5, L)$ :

$$
V=\left\{\left(\begin{array}{ccccc}
x & 0 & 0 & a_{1} & a_{2} \\
0 & 1 & \alpha & a_{3} & a_{4} \\
0 & 0 & 1 & 0 & \beta \\
0 & 0 & 0 & y & a_{5} \\
0 & 0 & 0 & 0 & 1
\end{array}\right) \quad \begin{array}{l}
(x, y) \in L^{*} \times L^{*}, \\
(\alpha, \beta) \in R \times R, \\
a_{i} \in L \text { for } i=1,2,3,4,5
\end{array}\right\}
$$

where, for every $\left(v_{1}, v_{2}\right) \in V \times V$, the product $v_{1} v_{2}$ is

$$
\left(\begin{array}{ccccc}
x_{1} x_{2} & 0 & 0 & x_{1} a_{1,2}+{ }_{L} a_{1,1} y_{2} & x_{1} a_{2,2}+{ }_{L} a_{1,1} a_{5,2}+{ }_{L} a_{2,1} \\
0 & 1 & \alpha_{1} \alpha_{2} & a_{3,2}+{ }_{L} a_{3,1} y_{2} & a_{4,2}+{ }_{L} \overline{\alpha_{1}} \beta_{2}+{ }_{L} a_{3,1} a_{5,2}+{ }_{L} a_{4,1} \\
0 & 0 & 1 & 0 & \beta_{1} \beta_{2} \\
0 & 0 & 0 & y_{1} y_{2} & y_{1} a_{5,2}+{ }_{L} a_{5,1} \\
0 & 0 & 0 & 0 & 1
\end{array}\right)
$$

where, for $i=1,2, v_{i}=\left(\begin{array}{ccccc}x_{i} & 0 & 0 & a_{1, i} & a_{2, i} \\ 0 & 1 & \alpha_{i} & a_{3, i} & a_{4, i} \\ 0 & 0 & 1 & 0 & \beta_{i} \\ 0 & 0 & 0 & y_{i} & a_{5, i} \\ 0 & 0 & 0 & 0 & 1\end{array}\right)$ and $\overline{\alpha_{i}}$ (resp. $\overline{\beta_{i}}$ ) is the image

of $\alpha_{i}$ (resp. $\beta_{i}$ ) in $L$. Then $V$ is a centerless connected interpretable solvable group and we have

$$
V^{\prime}=\left\{\left(\begin{array}{ccccc}
1_{L} & 0 & 0 & a_{1} & a_{2} \\
0 & 1 & 1_{K} & a_{3} & a_{4} \\
0 & 0 & 1 & 0 & 1_{K} \\
0 & 0 & 0 & 1_{L} & a_{5} \\
0 & 0 & 0 & 0 & 1
\end{array}\right) \mid a_{i} \in L \text { for } i=1,2,3,4,5\right\} .
$$

In particular, we obtain $V^{\prime}=\left[V, V^{\prime}\right]$. 
We consider $G=\left(K_{+} \times K_{+}\right) \rtimes V$ where $V$ acts by conjugation on $K_{+} \times K_{+}$such that, for any $(r, s) \in K_{+} \times K_{+}$and any $v \in V$, if $v=\left(\begin{array}{ccccc}x & 0 & 0 & a_{1} & a_{2} \\ 0 & 1 & \alpha & a_{3} & a_{4} \\ 0 & 0 & 1 & 0 & \beta \\ 0 & 0 & 0 & y & a_{5} \\ 0 & 0 & 0 & 0 & 1\end{array}\right)$ for $(x, y) \in L^{*} \times L^{*},(\alpha, \beta) \in R \times R$, and $a_{i} \in L$ for $i=1,2,3,4,5$, then $(r, s)^{v}=(\alpha r, \beta s)$. Thus we have $G^{\prime}=\left(K_{+} \times K_{+}\right) \times V^{\prime}$, so $G^{\prime}$ is the Fitting subgroup of $G$. Moreover, since $V$ is centerless, $G$ is centerless too.

Let $U$ and $T$ be two nilpotent subgroups of $G$ such that $U$ is normal in $G$ and $G=U T$. We will show that $T$ is nonabelian and that $U \cap T$ is infinite. First we show that $U=G^{\prime}$. Since $U$ is nilpotent, we have $U \leq F(G)=G^{\prime}$. Moreover, since $G / U \simeq T /(T \cap U)$ is nilpotent, $V /(V \cap U)$ is nilpotent too. Thus, since $V^{\prime}=\left[V, V^{\prime}\right]$, the subgroup $U$ contains $V^{\prime}$. Also, the action of $V$ on $K_{+} \times K_{+}$is transitive, so $V$ centralizes no nontrivial section of $K_{+} \times K_{+}$, and we have $\left[V, K_{+} \times K_{+}\right]=K_{+} \times K_{+}$, so $K_{+} \times K_{+}$ is contained in $U$. This proves that $U$ contains $G^{\prime}=\left(K_{+} \times K_{+}\right) \times V^{\prime}$, hence $U=G^{\prime}$, and $U$ is definable.

We show that we may assume $T \leq C$ for $C$ a Carter subgroup of $G$. Since $T$ is nilpotent, there is a nilpotent definable subgroup $T_{d}$ of $G$ containing $T$, and $T_{0}=$ $T \cap T_{d}^{\circ}$ has finite index in $T$, so $F(G) T_{0}$ has finite index in $G$. Since $G / F(G)$ is divisible (Fact 3.1), $G / F(G)$ has no proper subgroup of finite index, and we obtain $G=F(G) T_{0}=U T_{0}$. Therefore we may assume $T=T_{0}$, and $T$ is contained in a nilpotent connected definable subgroup. Now there exists a maximal nilpotent connected definable subgroup $C$ of $G$ containing $T$. Then $G=U C$, so we have $N_{G}(C)=N_{G^{\prime}}(C) C$ and, since $G^{\prime}$ is nilpotent, $N_{G}(C)$ is nilpotent. This implies $N_{G}(C)^{\circ}=C$, so $C$ is a Carter subgroup of $G$.

We consider the following nilpotent connected definable subgroup

$$
D=\left\{\left(\begin{array}{ccccc}
x & 0 & 0 & 0 & 0 \\
0 & 1 & \alpha & 0 & a \\
0 & 0 & 1 & 0 & \beta \\
0 & 0 & 0 & y & 0 \\
0 & 0 & 0 & 0 & 1
\end{array}\right) \mid(x, y) \in L^{*} \times L^{*},(\alpha, \beta) \in R \times R, a \in L\right\}
$$

Then $D$ is self-normalizing in $V$, that is $D$ is a Carter subgroup of $V$. We show that $C$ and $D$ are conjugate. Let $W_{1}=K_{+} \times\{0\}$ and $W_{2}=\{0\} \times K_{+}$. Then $W_{1}$ is a normal subgroup of $G$, and no nontrivial proper subgroup of $W_{1}$ is normal in $G$. Since $W_{2} V$ is a complement to $W_{1}$ in $G$, this shows that $W_{2} V$ is a maximal proper 
connected definable subgroup of $G$, and $W_{2} V$ contains a Carter subgroup of $G$ (Fact 4.1 (2)). Then, by Fact 4.1 (1), each Carter subgroup of $W_{2} V$ is a Carter subgroup of $G$. In the same way, $V$ is a maximal proper connected definable subgroup of $W_{2} V$, and each Carter subgroup of $V$ is a Carter subgroup of $G$, so $D$ is a Carter subgroup of $G$. Consequently, by Fact 4.1 (1), $C$ and $D$ are conjugate, and we may assume $D=C$ and $T \leq D$.

Now we consider the following central subgroup of $D$ :

$$
Z=\left\{\left(\begin{array}{ccccc}
1_{L} & 0 & 0 & 0 & 0 \\
0 & 1 & 1_{K} & 0 & a \\
0 & 0 & 1 & 0 & 1_{K} \\
0 & 0 & 0 & 1_{L} & 0 \\
0 & 0 & 0 & 0 & 1
\end{array}\right) \mid a \in L\right\}
$$

In particular we have $Z=D \cap V^{\prime}=D \cap U$ and $T Z=T(D \cap U)=D$. Then, if $T$ is abelian, $T Z$ is abelian too since $T \leq D$, which contradicts that $D$ is nonabelian, so $T$ is nonabelian. Thus we may assume that $U \cap T$ is finite. But $U \cap T \leq D \cap U=Z$ is central in $D$, and $T /(U \cap T) \simeq G / U$ is abelian. Hence, for each $t \in T$, the map $\operatorname{ad}_{t}: T \rightarrow U \cap T$, defined by $\operatorname{ad}_{t}(x)=[t, x]$ for each $x \in T$, is a homomorphism and $\operatorname{ad}_{t}(T)$ is a finite subgroup isomorphic to $T / C_{T}(t)$. Since $C_{T}(t)$ contains $U \cap T \leq Z(D)$ and since $T /(U \cap T) \simeq G / F(G)$ is divisible (Fact 3.1), the subgroup $\operatorname{ad}_{t}(T)$ is finite and divisible, so it is trivial. This proves that $T$ is abelian, contradicting that $T$ is nonabelian. This finishes the proof.

\section{Definability of complements}

In Proposition 3.7, we provided a centerless connected solvable group $G$ of finite Morley rank such that $F(G)$ has definable complements and nondefinable complements. In this section we show that $F(G)$ can have no definable complement (Proposition 5.1).

We will deduce from this result that some groups of finite Morley rank are not definably linear. A group $G$ of finite Morley rank is definably linear (over finitely many interpretable fields $K_{1}, \ldots, K_{n}$ ), if it has an interpretable faithful linear representation over the ring $K_{1} \oplus \cdots \oplus K_{n}$ (Proposition 5.3 and Corollary 5.4).

Proposition 5.1 There exists a centerless connected solvable group $G$ of finite Morley rank such that, for each normal nilpotent subgroup $U$ and each definable nilpotent subgroup $T$, the equality $G=U T$ implies that $U \cap T$ is infinite. 
Furthermore, we may choose $G$ to be isomorphic as abstract group with a connected algebraic group over an algebraically closed field.

Proof Let $K$ be an algebraically closed field of characteristic zero. We assume that $K$ is not isomorphic to $\overline{\mathbb{Q}}$, namely $K$ has a nonzero derivation $\delta$. We consider the group

$$
G=\left\{\left(\begin{array}{cccc}
t & a & b & u \\
0 & 1 & c & v \\
0 & 0 & t & w \\
0 & 0 & 0 & 1
\end{array}\right) \mid t \in K^{*},(a, b, c, u, v, w) \in K^{6}\right\} .
$$

Then $G$ is a centerless connected group of Morley rank exactly 7 as an algebraic group, and of Morley rank at most 7 as a group. We consider a decomposition of $G$ of the form $G=U T$ for a normal nilpotent subgroup $U$ and a definable nilpotent subgroup $T$, and we show that $U \cap T$ is infinite.

As usual, $U=F(G)$, the group of strictly upper triangular matrices. In particular $U$ is torsion-free, so we may assume that $G=U \rtimes T$. But definable subgroups of $G$ are Zariski closed, so $T$ is a closed subgroup of $G$. Hence, since $U$ is the unipotent radical of $G$ and since $G=U \rtimes T$, the subgroup $T$ is a maximal torus of $G$.

By conjugacy of maximal tori in $G$, we may assume that $T$ is the diagonal subgroup of $G$. Let $\alpha: G \rightarrow G$ be the map defined by

$$
\alpha\left(\begin{array}{cccc}
t & a & b & u \\
0 & 1 & c & v \\
0 & 0 & t & w \\
0 & 0 & 0 & 1
\end{array}\right)=\left(\begin{array}{cccc}
t & a & b+\delta(t) & u+\delta(w) \\
0 & 1 & c & v \\
0 & 0 & t & w \\
0 & 0 & 0 & 1
\end{array}\right) .
$$

It is an automorphism of the pure group $G$, so it preserves its definable subsets. Thus $\alpha(T)$ and $T \cap \alpha(T)$ are definable in the pure group $G$. Since the pure group $G$ is interpretable in the pure field $K$, this implies that $T \cap \alpha(T)$ is definable in the pure field $K$ too, that is $T \cap \alpha(T)$ is a closed subgroup. But we have

$$
T \cap \alpha(T)=\left\{\left(\begin{array}{cccc}
t & 0 & 0 & 0 \\
0 & 1 & 0 & 0 \\
0 & 0 & t & 0 \\
0 & 0 & 0 & 1
\end{array}\right) \mid t \in K^{*}, \delta(t)=0\right\},
$$

and $\delta$ is a nonzero derivation. Hence $T \cap \alpha(T)$ is a proper closed subgroup of $T$, and since $T$ is a torus of dimension one over $K$, the subgroup $T \cap \alpha(T)$ is finite. This yields the final contradiction since $\delta(t)=0$ for each element of the prime subfield of $K$. 
It is noticeable that the group considered in the previous proof is not definably linear, although it is centerless and connected (Proposition 5.3). The proof uses the fact below.

Fact 5.2 [9, Théorème 4.15] Let $F$ be an algebraically closed field. Then, in the pure field $F$, every infinite definable field $K$ is definably isomorphic to $F$.

Proposition 5.3 There exists a centerless connected solvable group of finite Morley rank which is not definably linear.

Furthermore, we may choose this group to be isomorphic as abstract group with a connected algebraic group over an algebraically closed field.

Proof Let $K, G, T$ and $\alpha$ be as in the proof of Proposition 5.1. We assume toward a contradiction that $G$ is definably linear over finitely many interpretable fields $K_{1}, \ldots, K_{n}$. Then there exist $i \in\{1, \ldots, n\}$ and a definable representation $\gamma$ of $G$ over $K_{i}$ such that the kernel $C$ of $\gamma$ does not contain the subgroup

$$
Z(F(G))=\left\{\left(\begin{array}{llll}
1 & 0 & 0 & u \\
0 & 1 & 0 & 0 \\
0 & 0 & 1 & 0 \\
0 & 0 & 0 & 1
\end{array}\right) \mid u \in K\right\} .
$$

But $Z(F(G))$ is the only minimal nontrivial normal closed subgroup of $G$, so $C=1$. Hence $\gamma$ is an interpretable embedding from $G$ to an algebraic group $H$ over $K_{i}$.

By Fact 5.2, there is a field isomorphism $\varphi: K_{i} \rightarrow K$, definable in the pure field $K$. So the corresponding map $\varphi^{0}: H \rightarrow{ }^{\varphi} H$ is definable in $K$, where ${ }^{\varphi} H$ denotes the algebraic group over $K$ obtained by transfer of base field. In particular, for each subset $X$ of $H$, the set $X$ is Zariski closed in $H$ if and only if $\varphi^{\circ}(X)$ is Zariski closed in ${ }^{\varphi} H$. In other words, $X$ is definable in $K_{i}$ if and only if $\varphi^{\circ}(X)$ is definable in $K$. Since $\gamma$ is interpretable in $G$, which is an algebraic group over $K$, it is interpretable in $K$, and since $T$ is a Zariski closed subgroup of $G$, the subgroup $\gamma(T)$ of $H$ is definable in $K$. Thus $\varphi^{\circ}(\gamma(T))$ is definable in $K$ too, and $\gamma(T)$ is definable in $K_{i}$. Since $K_{i}$ and $\gamma$ are interpretable in $G$, we obtain the definability of $T$ in $G$. The latter contradicts the proof of Proposition 5.1, which says that $T$ is not definable in $G$. Thus $G$ is not definably linear, as desired.

In particular, Proposition 5.3 provides a counterexample to [6, Conjecture 1.3]. Indeed, the notion of geometric group was introduced in [6] as follows: 
(1) $\left[6\right.$, Definition 1.5 and Remark 1.6] if, for $I$ an interpretable set, $\mathscr{F}=\left\{F_{i} \mid i \in I\right\}$ is a uniformly definable family of connected definable subgroups of a group $G$ of finite Morley rank, then $\mathscr{F}$ is said to be geometric when $\left\{g \in G \mid \exists ! i \in I, g \in F_{i}\right\}$ forms a generic subset of $G$;

(2) [6, Definition 1.10] a group $G$ of finite Morley rank is said to be geometric when, for each distinct elements $x$ and $y$ of $G$, there exists a geometric family $\mathscr{F}$ of subgroups of $G$ such that $x y^{-1} \notin \cup \mathscr{F}$.

Then [6, Conjecture 1.3] states that any geometric group of finite Morley rank is definably linear. Moreover, if this last conjecture holds, then any centerless connected group of finite Morley rank would be definably linear by [6, Théorème 4.18 and Corollaire 2.9]. But this fails by Proposition 5.3, hence we obtain the following result.

Corollary 5.4 There exists a geometric group of finite Morley rank which is not definably linear.

\section{Acknowledgments}

I thank Tuna Altınel very much for his questions concerning the first version of this work. They permitted a substantial improvement of this article.

\section{References}

[1] T Altınel, J S Wilson, On the linearity of torsion-free nilpotent groups of finite Morley rank, Proc. Amer. Math. Soc. 137 (2009), 1813-1821; doi: 10.1090/S0002-9939-0809695-0

[2] A Baudisch, M Hils, A Martin-Pizarro, F O Wagner, Die böse Farbe, J. Inst. Math. Jussieu 8 (2009), 415-443; doi:10.1017/S1474748008000091.

[3] A V Borovik, A Nesin, Groups of finite Morley rank, volume 26 of Oxford Logic Guides, Clarendon Press (OUP), Oxford, 1994.

[4] O Frécon, Sous-groupes anormaux dans les groupes de rang de Morley fini résolubles, J. Algebra 229 (2000) 118-152; doi:10.1006/jabr.2000.8302.

[5] O Frécon, Sous-groupes de Hall généralisés dans les groupes résolubles de rang de Morley fini, J. Algebra 233 (2000), 253-286; doi:10.1006/jabr.2000.8437.

[6] O Frécon, Groupes géométriques de rang de Morley fini, J. Inst. Math. Jussieu 7 (2008), 751-792; doi:10.1017/S1474748008000212. 
[7] O Frécon, Pseudo-tori and subtame groups of finite Morley rank, J. Group Theory 12 (2009), 305-315; doi:10.1515/JGT.2008.079.

[8] A Nesin, On solvable groups of finite Morley rank, Trans. Amer. Math. Soc. 321 (1990), 659-690; doi:10.2307/2001580.

[9] B Poizat, Groupes stables, Bruno Poizat, Lyon (1987).

[10] B Poizat, MM. Borel, Tits, Zil' ber et le Général Nonsense, J. Symbolic Logic 53 (1988), 124-131; doi:10.2307/2274432.

[11] F O Wagner, Nilpotent complements and Carter subgroups in stable $\Re$-groups, Arch. Math. Logic 33 (1994), 23-34: doi:10.1007/BF01275468.

[12] F O Wagner, Fields of finite Morley rank, J. Symbolic Logic 66 (2001), 703-706; doi: $10.2307 / 2695038$.

Laboratoire de Mathématiques et Applications, Université de Poitiers, Téléport 2 - BP 30179,

Boulevard Marie et Pierre Curie, 86962 Futuroscope Chasseneuil Cedex, France

olivier.frecon@math.univ-poitiers.fr

Received: 11 May 2010 Revised: 16 June 2010 